$621.43 .06: 532.595$

\title{
Production of Negative Pressure by an Exhaust Pipe with Stepped or Conical Open End*
}

\author{
By Fujio NaGao** and Yuzuru Shimamoto***
}

\begin{abstract}
It is a well known fact that the scavenging of an internal combustion engine by the utilization of the wave effect in the exhaust pipe is promoted by a stepped or conical open end. However, most of the works along this line hitherto performed are confined to the qualitative ones. The authors have theoretically treated the wave phenomena in the pipe with a stepped or conical open end, and determined the optimum area-ratio of the step or the diffuser to produce the maximum energy of rarefaction wave. The results obtained are experimentally confirmed with an air model by measuring the energy of rarefaction wave.
\end{abstract}

\section{Introduction}

The pressure fluctuation in the exhaust system affects considerably the engine performance. Twocycle engines are particularly sensitive to the exhaust pressure fluctuations because they depend on the pressure gradient ra her than on the piston motion for the scavenging of cylinders. It is a well known fact that the scavenging is promoted by the utilization of the pressure wave in the exhaust pipe and the adoption of the exhaust pipe with a stepped or conical open end brings the more desirable results. Many studies along this line have already been carried out ${ }^{(1)-(5)}$. However, the quantitative one on the effect of the stepped or conical open end seems to have hardly ever been tried. The pressure wave in the exhaust pipe consists of two kinds of waves. One propagates from the engine side to the open end and the other in the opposite direction. The scavenging is helped by the rarefaction wave, which is produced by the reflection of compression wave propagating toward the open end. Therefore, by the energy ratio between the compression wave and the corresponding reflection wave, it can be estimated how effectively the compression wave produced on the engine side is reflected to promote the scavenging as the rarefaction wave. The authors have investigated the effect of the stepped or conical open end by measuring the energy of pressure wave in an air model. To simplify the measurement of energy, the

* Received 20th March, 1958.

** Professor, Department of Mechanical Engineering, Kyoto University, Sakyo-ku, Kyoto.

*** Student of Post-graduate Course, Kyoto University. exhaust pipe is made so long that the pressure changes of the compression wave and its reflection wave can separately be recorded.

\section{Theoretical treatment}

It is assumed that the flow in the exhaust pipe is a one-dimensional one. Ignoring the friction loss and the change of entropy, and using an average value of entropy, the characteristic equations in the exhaust pipe ${ }^{(6)}$ are given by

$$
\begin{aligned}
& \frac{d x}{d\left(t a_{0}\right)}=U \pm P \ldots \ldots \ldots \ldots \ldots \ldots \ldots \ldots \ldots \ldots \\
& d U=\mp \alpha d P \mp P U \frac{d \log f}{d x} d\left(t \dot{a_{0}}\right)
\end{aligned}
$$

where $a_{0}$ is the sonic velocity for reference, $f$ the cross-sectional area of the exhaust pipe, $p$ the pressure, $p_{0}$ the pressure for reference (the atmospheric pressure), $P=\left(p / p_{0}\right)^{(\kappa-1) / 2 \kappa}, t$ the time, $u$ the velocity, $U=u / a_{0}, x$ the distance measured along the exhaust pipe, $\alpha=2 /(\kappa-1)$, and $\kappa$ the ratio of specific heats.

The state in the exhaust pipe is given by solving Eqs. (1) and (2) with the boundary conditions.

The energy of pressure wave $E$ passing a section of the exhaust pipe is obtained by integrating the product of the effective energy and the flow rate of gas with respect to the time $t$.

$$
E=\frac{\kappa f a_{0} p_{0}}{2} \int|U| P^{\alpha}\left\{U^{2}+\alpha\left|P^{2}-1\right|\right\} d t
$$

Under the condition that the cross-sectional area of the exhaust pipe is constant and the pressure wave propagates in only one direction, the relation between the pressure and the velocity of pressure wave is represented by 


$$
U= \pm \alpha(P-1)
$$

By introducing this equation into Eq. (3), we obtain

$$
E=\frac{\alpha(\alpha+2)}{2} f a_{0} p_{0} \int P^{\alpha}(P-1)^{2}(\alpha|P-1|+P+1) d t
$$

By assuming that the pressure at the open end of the exhaust pipe is equal to the atmospheric pressure, the end condition is given as follows :

$$
P=1
$$

Since the time lag existing between the states on both sides of the step is negligibly small compared with the duration of pressure fluctuation, the equation of steady flow may be approximately applicable to that at the step. Then the boundary condition at the step is given by combining the equations of the steady flow with the characteristic equations in the exhaust pipes on both sides of the step. In the case that gas flows from the small cross-sectional side of the step to the other as shown in Fig. 1 and the velocity at the step is subsonic, the boundary condition is obtained in the following way.

By transforming the equation of continuity at the step to a non-dimensional form, we obtain

$$
P_{2}{ }^{\alpha} U_{2}=\varphi P_{3}{ }^{\alpha} U_{3} \cdot a_{0} / a_{0}{ }^{\prime}
$$

where $a_{0}{ }^{\prime}$ is the sonic velocity for reference in the exhaust pipe on the large cross-sectional side of the step, $U_{3}=u_{3} / a_{0}{ }^{\prime}$, and $\varphi$ the area-ratio $\left(f_{3} / f_{2}\right)$. Suffixes 2 and 3 refer to the states on the small and large cross-sectional sides of the step respectively. The sonic velocity for reference on the large cross-sectional side of the step is not equal to that on the other side due to the change of entropy.

The equations of momentum and energy may be written as follows :

$$
\begin{aligned}
& \kappa P_{2}{ }^{\alpha} U_{2}{ }^{2}+\varphi P_{2}{ }^{\alpha+2}=\kappa \varphi P_{3}{ }^{\alpha} U_{3}{ }^{2}+\varphi P_{3}{ }^{\alpha+2} \\
& \alpha P_{2}{ }^{2}+U_{2}{ }^{2}=\left(\alpha P_{3}{ }^{2}+U_{3}{ }^{2}\right)\left(a_{0}{ }^{\prime} / a_{0}\right)^{2} .
\end{aligned}
$$

The characteristic equations in the exhaust pipes are given by integrating Eq. (2) under the condition of $d \log f / d x=0$. The characteristic equation on the large cross-sectional side of the step must be calculated by using $a_{0}{ }^{\prime}$ instead of $a_{0}$.

$$
\begin{aligned}
& \alpha\left(P_{1}-P_{2}\right)=U_{2} . \\
& \alpha\left(P_{3}-P_{4}\right)=U_{3} .
\end{aligned}
$$

where suffixes 1 and 4 refer to the states under the condition of $U=0$.

By eliminating $a_{0}{ }^{\prime} / a_{0}, U_{2}$ and $U_{3}$ from Eqs. $(7) \sim(11)$, and dividing by $P_{1}$, we have

$$
\begin{aligned}
& \alpha(\alpha+2)\left(1-\frac{P_{2}}{P_{1}}\right)^{2}\left(\frac{P_{2}}{P_{1}}\right)^{\alpha}+\varphi\left(\frac{P_{2}}{P_{1}}\right)^{\alpha+2}=\alpha(\alpha+2) \varphi\left(\frac{P_{3}}{P_{1}}-\frac{P_{4}}{P_{1}}\right)^{2}\left(\frac{P_{3}}{P_{1}}\right)^{\alpha}+\varphi\left(\frac{P_{3}}{P_{1}}\right)^{\alpha+2} \ldots \\
& \left\{\left(\frac{P_{2}}{P_{1}}\right)^{2}+\alpha\left(1-\frac{P_{2}}{P_{1}}\right)^{2}\right\}\left(\frac{P_{2}}{P_{1}}\right)^{2 \alpha}\left(1-\frac{P_{2}}{P_{1}}\right)^{2}=\varphi^{2}\left\{\left(\frac{P_{3}}{P_{1}}\right)^{2}+\alpha\left(\frac{P_{3}}{P_{1}}-\frac{P_{4}}{P_{1}}\right)^{2}\right\}\left(\frac{P_{3}}{P_{1}}\right)^{2 \alpha}\left(\frac{P_{3}}{P_{1}}-\frac{P_{4}}{P_{1}}\right)^{2} .
\end{aligned}
$$

Solving Eqs. (12) and (13), and putting $\alpha=5(\kappa=1.4)$, the pressures $P_{2} / P_{1}$ and $P_{3} / P_{1}$ are obtained for the given values of $P_{4} / P_{1}$ and $\varphi$ as shown in Fig. 2. When pressure waves propagating toward the step from both its sides reach there at the same time, the characteristic lines of those waves are traced in the state diagram of Fig. 3. The values of $P_{1}$ and $P_{4}$ can be given by the intersections of those lines with $P$-axis. By applying the value of $P_{4} / P_{1}$ thus obtained, the values $P_{2} / P_{1}$ and $P_{3} / P_{1}$ are easily found out for the given value of $\varphi$ as shown in Fig. 2. The points, corresponding to $P_{2}$ and $P_{3}$ on the above mentioned characteristic lines in Fig. 3, represent the states on both sides of the step respectively.

In order to represent the effectiveness of the exhaust pipe in producing the rarefaction wave,

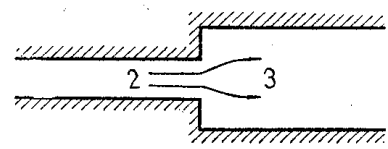

Fig. 1

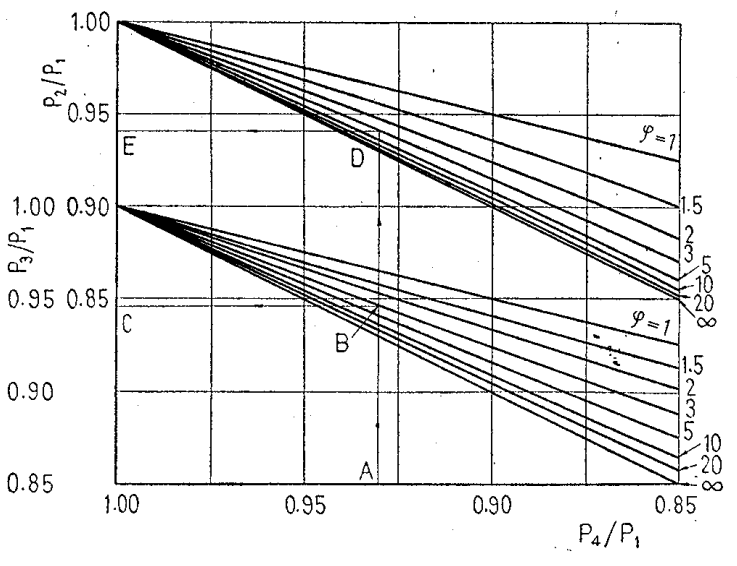

Fig. 2 Boundary condition at a step 


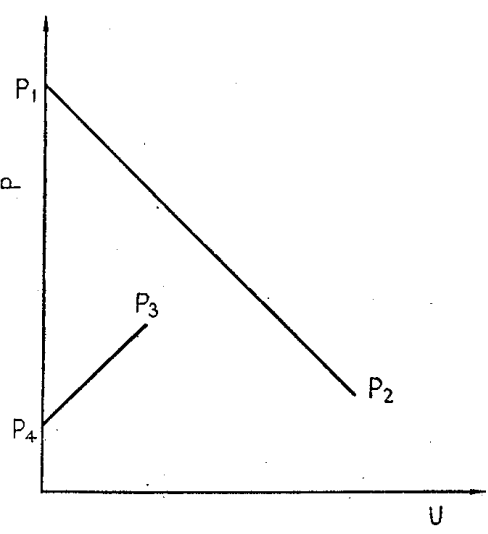

Fig. 3 State diagram

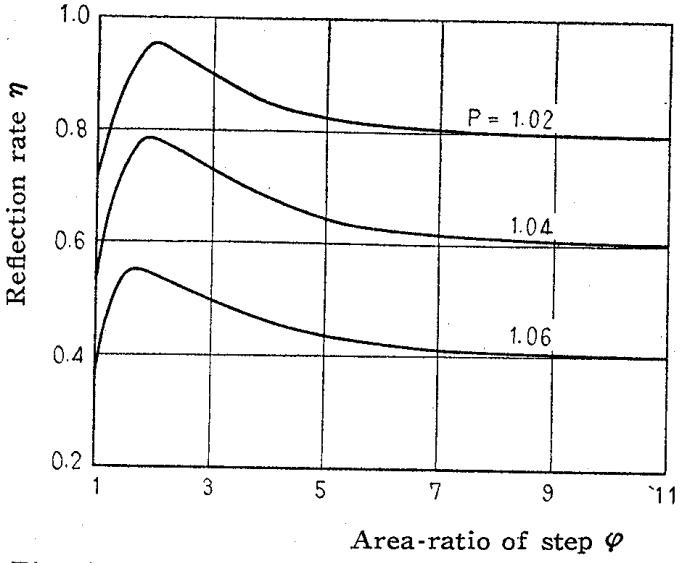

Fig. 4 Reflection rate of energy in exhaust pipe with a stepped open end (In case of $2 L / a_{0} T=0$ )

we used the reflection rate of energy $\eta$ defined as follows :

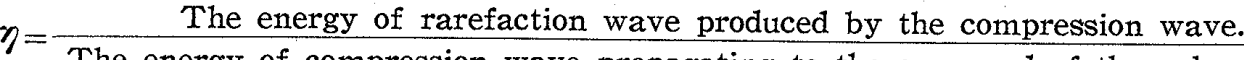

\section{(a) The exhaust pipe with a stepped open end}

Main factors affecting the reflection rate of energy of the compression wave, which propagates toward the open end, seem to be the pressure of compression wave $p$, the duration $T$ required for the compression wave to pass a section, the area-ratio of the step $\varphi$ and the length of the exhaust pipe $L$ between the step and the open end. Those factors may be reduced to three non-dimensional forms, i.e. $P, \varphi$ and $2 L / a_{0} T$. The pressure waves reflected at the open end and the step are separated or entirely overlapped according as $2 L / a_{0} T=1$ or 0 . Therefore, the usual state of reflection waves can be estimated from both extreme states of $2 L / a_{0} T=0$ and 1 .

In the case of $2 L / a_{0} T=0$, the boundary condition at the step can be represented by combining with that at the open end. By putting $P_{3}=1$, and eliminating $a_{0}{ }^{\prime} / a_{0}$ and $U_{3}$ from Eqs. (7), (8), and (9), we get

$$
\alpha\left(P_{2}{ }^{\alpha+2}-1\right)\left(P_{2}{ }^{\alpha+2}+\alpha+1\right) \varphi^{2}+(\alpha+2) P_{2}{ }^{\alpha}{U_{2}}^{2}\left(2 P_{2}{ }^{\alpha+2}+\alpha\right) \varphi-(\alpha+2)^{2} P_{2}{ }^{2 \alpha+2} U_{2}{ }^{2}=0
$$

Considering that the compression wave propagating toward the open end consists of the elementary rectangular waves of various pressures, this elementary wave is taken up to investigate the effect of the :stepped open end. The rarefaction wave produced by the reflection of compression wave requires shorter time to pass a section than the compression wave. When the elementary wave reaches the step, its pressure $P$ and velocity $U$ are changed to $P_{2}$ and $U_{2}$ respectively, and the rarefaction wave of the pressure $P^{\prime}$ and the velocity $U^{\prime}$ are reflected. The ratio of the duration of reflection wave to pass a section to that of incident wave, may be simply assumed as follows :

$$
\frac{1 /\left(P^{\prime}-U^{\prime}\right)+1 /\left(P_{2}+U_{2}\right)}{1 /(P+U)+1 /\left(P_{2}-U_{2}\right)}
$$

An example of calculation for $\kappa=1.4$ is shown in Fig. 4 . With the increase in the pressure of elementary wave, the reflection rate becomes smaller, however the ratio of the energy recovered by the step to that reflected at the open end of the plain exhaust pipe $(\varphi=1)$ becomes larger. In the case that the sum of the loss at the step due to the enlargement of cross-section and the kinetic energy discharged into the atmosphere becomes minimum, the maximum reflection rate is obtained. The optimum area-ratio which makes the reflection rate maximum is obtained when the pressure at the step satisfying the boundary condition for the given value of $P$ is minimum. The relation between the incident wave and the state at the step is given by

$$
U_{2}-U=\alpha\left(P-P_{2}\right)
$$

By eliminating $U$ and $U_{2}$ from Eqs. (4), (14), and (15), differentiating by $\varphi$, and putting $d P_{2} / d \varphi=0$, we get

$$
2\left(P_{2}{ }^{\alpha+2}-1\right)\left(P_{2}{ }^{\alpha+2}+\alpha+1\right) \varphi+\alpha(\alpha+2) P_{2}{ }^{\alpha}\left(2 P_{2}{ }^{\alpha+2}+\alpha\right)\left(2 P-P_{2}-1\right)^{2}=0
$$

Solving simultaneously Eq. (16) and Eq. (14), the optimum area-ratio is obtained. As shown in Fig. 5, the optimum area-ratio and the maximum reflection rate become smaller with the increase in the pressure of 


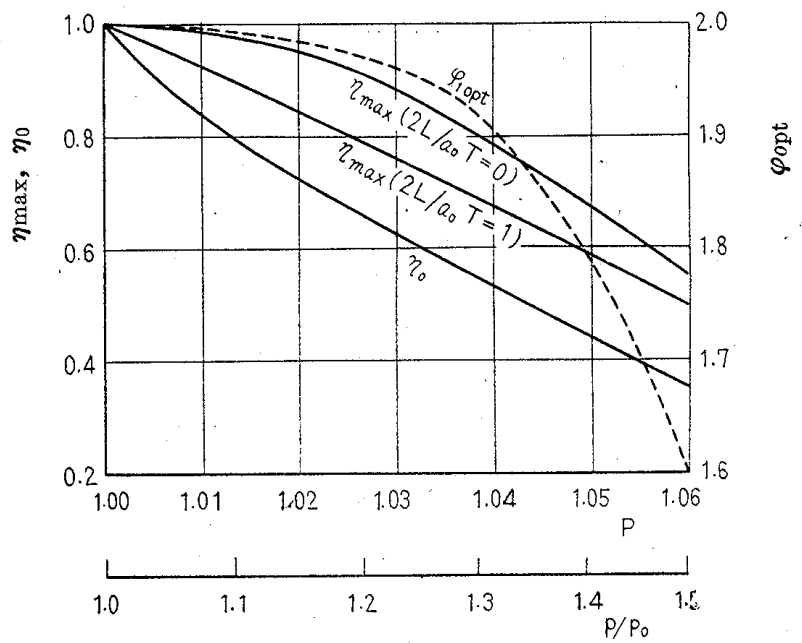

Fig. 5 Optimum area-ratio of step and maximum reflection rate

elementary wave; $\eta_{0}$ in the same figure is the reflection rate at the open end of the plain exhaust pipe.

In the case of $2 L / a_{0} T=1$, the reflection rate is calculated by using the sum of the energies of reflection waves at the step and the open end.

The ratio of the duration of reflection wave to pass a section to that of the incident wave, may be assumed in the same way as in the case of $2 L / a_{0} T$ $=0$. As shown in Fig. 6, there exists the optimum area ratio which makes the reflection rate maximum. The loss at the step due to the enlargement of cross-section is smaller than that in the case of $2 L / a_{0} T=0$ because of the smaller pressure difference between both sides of step. Therefore, the optimum area-ratio, which makes the sum of the loss at the step and the kinetic energy discharged into the atmosphere minimum, becomes larger than that in the case of $2 L / a_{0} T=0$. However, as the kinetic energy discharged increases and a part of the rarefaction wave reflected at the open end is reflected again at the step toward the open end, the maximum reflection rate is small compared with that in the case of $2 L / a_{0} T=0$ as shown in Fig. 5. The optimum area-ratio lies between 2 and 3 , and decreases with the increase in the pressure of wave.

In the case that the compression wave of a sine pattern propagates to the open end, the reflection rate is calculated with the characteristic method by using Eqs. (1) and (2). Fig. 7 shows the results obtained for the following sine wave:

$$
\left.\begin{array}{rrrr}
P=1+0.05 \sin \frac{\pi}{T} t, U & =0.25 \sin \frac{\pi}{T} t & \text { for } 0 \leqq t \leqq T \\
P=1, & U=0 & \text { for } t>T
\end{array}\right\}
$$

When the shock wave occurs in the exhaust pipe,

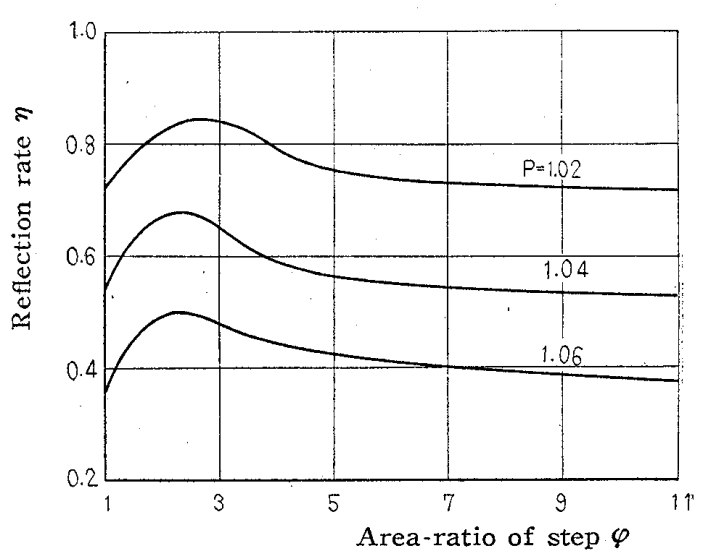

Fig. 6 Reflection rate of energy in exhaust pipe with a stepped open end (In case of $2 L / a_{0} T=1$ )

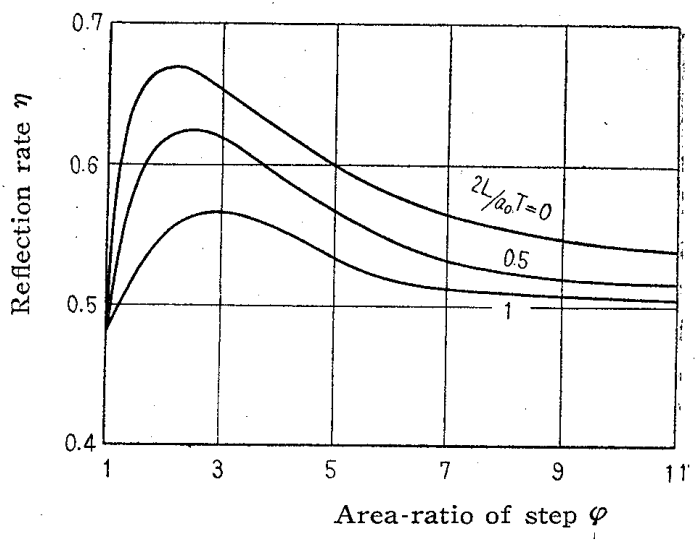

Fig. 7 . Refiection rate of energy in exhaust pipe with a stepped open end. (In case of compression wave of sine pattern)

the calculation is carried out under the assumptiom. that the relation between the states before and. behind the shock wave can be represented by Eq. (2) and the propagating velocity of shock wave is. equal to the average value of the propagating velocities at the states before and behind the shock. wave. The reflection rate at the stepped open end. becomes maximum when the area-ratio is between. 1.5 and 3 , and becomes larger with the decreasein the length of exhaust pipe between the step and. the open end.

\section{(b) The exhaust pipe with a conical open end}

The energy losses in the exhaust pipe with a: conical open end are the kinetic energy discharged into the atmosphere, the friction loss and the loss. due to the shock wave. The kinetic energy discharged decreases with the increase in the arearatio $\varphi$ of the diffuser to its inlet. However, the area-ratio must be decided in consideration of other. losses. Using Eqs. (1) and (2), the reffection rateof the compression wave given by Eq. (17) is calculated as shown in Fig. 8. It can be seen that: 


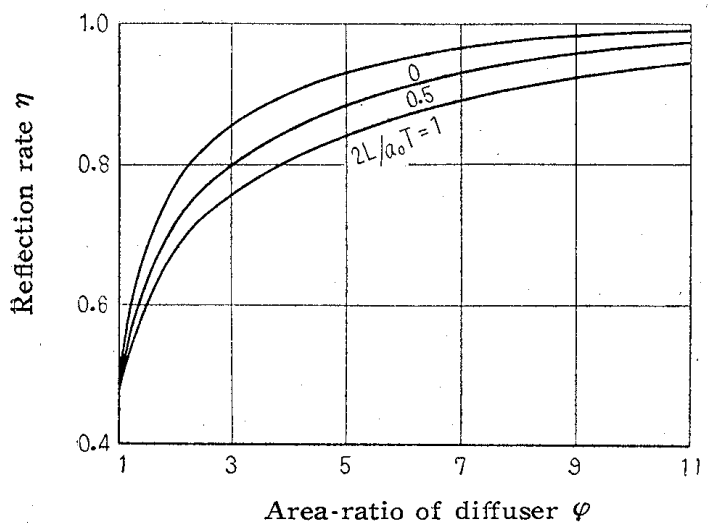

Fig. 8 Reflection rate of energy in exhaust pipe with a conical open end (In case of compression wave of sine pattern)

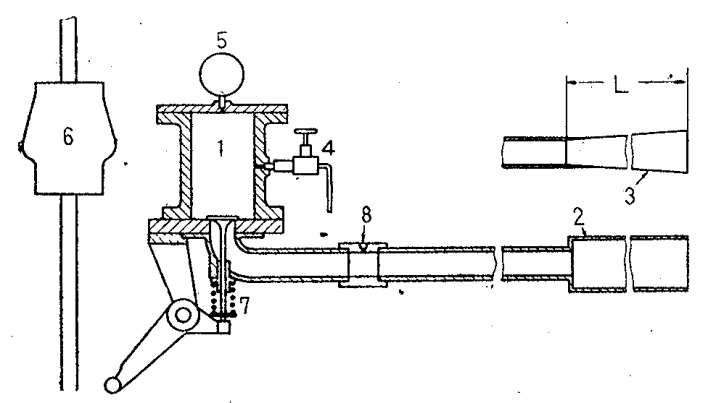

1. Cylinder $\left(2045 \mathrm{~cm}^{3}\right), 2$. Exhaust pipe with a stepped open end, 3. Exhaust pipe with a conical open end, 4. Valve, 5. Pressure gauge 6 . Falling weight, 7. Exhaust valve, 8. Connection for indicator

Fig. 9 Schema of experimental apparatus

the reflection rate at the conical open end is considerably larger than that at the stepped open end and the degree of improvement of the reflection rate becomes smaller with the increase in the arearatio.

\section{Experimental apparatus and methods}

Fig. 9 is the schema of the experimental apparatus, which is made by decreasing the cylinder volume of the apparatus already reported( ${ }^{(7)}$ and fitting the exhaust pipe with a stepped or conical open end. The pressure of air supplied into the cylinder through the valve is measured by the pressure gauge. Opening the exhaust valve with the falling weight, the air in the cylinder is ejected into the exhaust pipe. The pressure changes of the compression wave and its reflection wave thus produced in the exhaust pipe are recorded by an indicator of photo-electric type. The length of exhaust pipe between the step and the open end, and that of conical pipe are 0.5 or $1.7 \mathrm{~m}$. The duration of the exhaust valve opening is $0.034 \mathrm{sec}$. To make the reflection wave reach the exhaust

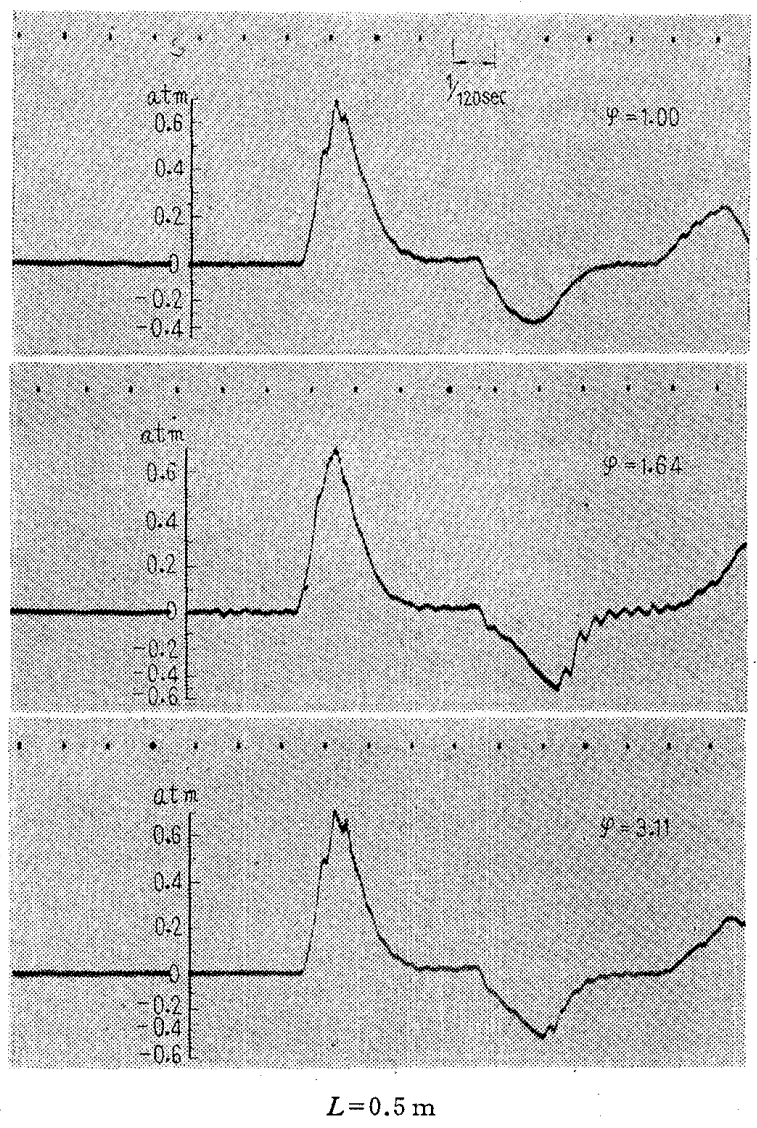

Fig. 10 Pressure changes in exhaust pipe with a stepped open end

valve after its closing and to record the pressure changes of the compression wave and its reflection wave separately, the length of exhaust pipe between the exhaust valve and the step or the inlet of the diffuser is chosen $5.6 \mathrm{~m}$. The maximum pressure of compression wave produced are 0.7 and 0.45 atm for the initial cylinder pressure of 3.0 and 1.9 atm respectively.

\section{Experimental results}

Fig. 10 is a typical example of the pressure recorded. The energy of compression wave can be calculated with Eq. (5). When the negative reflection wave reaches the position of indicator, the exhaust valve has already been closed. Assuming that the pressure recorded is the one at the closed end $P_{s}$, the pressure $P$ of reflection wave is given by the following equation,

$$
P=\frac{1}{2}\left(P_{s}+1\right)
$$

As the position of indicator is only $268 \mathrm{~mm}$ apart from the exhaust valve, the error due to the above mentioned assumption will be slight.

The effect of the stepped open end is shown in Figs. 11 and 12 by the ratio $\eta / \eta_{0}$ of the reflection 


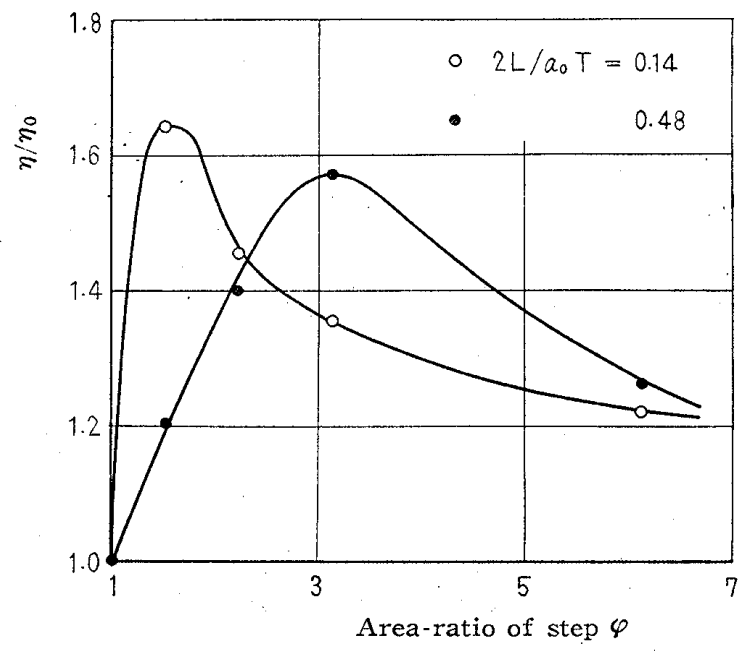

Maximum pressure of compression wave $0.7 \mathrm{~atm}$

Fig. 11 Reflection rate of energy in exhaust pipe with a stepped open end

rate at the stepped open end to that at the open end of the plain pipe. The ratio of the reflection rates becomes larger with the increase in the maximum pressure of compression wave. The arearatio making the ratio of the reflection rates maximum, agrees approximately with the optimum arearatio calculated. In the case that $2 L / a_{0} T$ is small, the reflection rate is considerably sensitive to the area-ratio.

Fig. 13 shows the effect of the conial open end. It is clearly seen that the effect of the conical open end is superior to that of the stepped open end.

\section{Conclusions}

By measuring the energy of negative reflection wave, which can be utilized for the scavenging, the effect of the exhaust pipe with a stepped or conical open end is investigated. The results obtained are summarized as follows. The energy of reflection wave becomes larger in the order of the plain pipe, the exhaust pipe with a stepped open end and one with a concial open end. The effect of the exhaust pipe with a stepped open end becomes more sensitive to the area-ratio with the decrease in the length of exhaust pipe between the step and the open end, and there is the optimum area-ratio that makes the negative reflection wave maximum. This optimum area-ratio seems to be between 1.5 and 3. Concerning the exhaust pipe with a conical open end, it is desirable to make the area-ratio large, however the degree of improvement of the

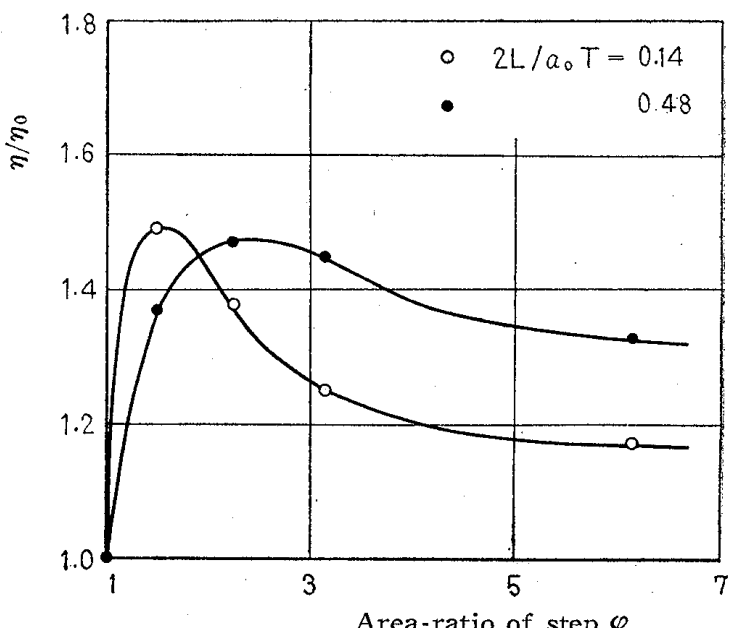

Maximum pressure of compression wave $0.45 \mathrm{~atm}$

Fig. 12 Reflection rate of energy in exhaust pipe with a stepped open end

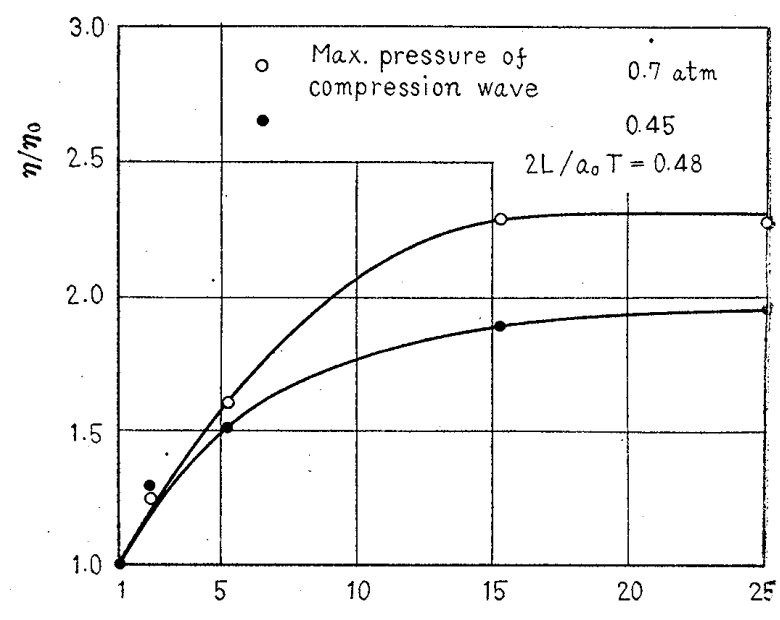

Area-ratio of diffuser $\varphi$

Fig. 13 Reflection rate of energy in exhaust pipe with a conical open end

reflection rate becomes smaller with the increase in the area-ratio.

\section{References}

(1) H.O. Farmer: Proc. Inst. Mech. Engrs., Vol. 138 (1938), p. 367.

(2) S.J. Davis : Engneermg., Vol. 143 (1937), p. 685 \& 715; Vol. 149 (1940), p. 515.

(3) F.J. Wallace and M.H. Nassif: Proc. Inst. Mech. Engrs:, Vol. 168 (1954), p. 515.

(4) F.J. Wallace: Engineermg., Vol. 178 (1954), p. 524.

(5) F. J. Wallace and G. Boxer: Proc. Inst. Mech. Engrs., Vol. 170 (1956), p. 1131.

(6) E. Jenny: Brown Boveri Review, Vol. 37, No. 11 (1950), p. 477 .

(7) F. Nagao and Y. Shimamoto: Bulletin of JSME, Vo1. 2, No. 5 (1959), p. 170. 